\title{
On the Zeros of Degree One $L$-functions from the Extended Selberg Class
}

\author{
Haseo Ki and Yoonbok Lee
}

March 18, 2010

\begin{abstract}
We investigate the zeros of degree one $L$-functions from the extended Selberg class off the critical line.
\end{abstract}

\section{Introduction}

In [14], Selberg introduced the class $\mathcal{S}$ consists of the functions $F(s)$ satisfying the following conditions.

1. (Dirichlet series) For $\sigma>1, F(s)$ is an absolutely convergent Dirichlet series

$$
F(s)=\sum_{n=1}^{\infty} \frac{a(n)}{n^{s}} \quad(s=\sigma+i t) .
$$

2. (Analytic continuation) For some integer $m \geqslant 0,(s-1)^{m} F(s)$ is an entire function of finite order.

3. (Functional equation) $F(s)$ satisfies a functional equation of the form

$$
\Phi(s)=\omega \bar{\Phi}(1-s),
$$

where

$$
\Phi(s)=Q^{s} \prod_{j=1}^{r} \Gamma\left(\lambda_{j} s+\mu_{j}\right) F(s)
$$

with $\bar{\Phi}(s)=\overline{\Phi(\bar{s})}, Q>0, \lambda_{j}>0, \operatorname{Re} \mu_{j} \geqslant 0$ and $|\omega|=1$. 
4. (Ramanujan hypothesis) For every $\epsilon>0, a(n) \ll n^{\epsilon}$.

5. (Euler product) For $\sigma$ sufficiently large,

$$
\log F(s)=\sum_{n=1}^{\infty} \frac{b(n)}{n^{s}} \quad(s=\sigma+i t),
$$

where $b(n)=0$ unless $n$ is a positive power of a prime, and $b(n) \ll n^{\theta}$ for some $\theta<\frac{1}{2}$.

For a function $F(s)$ in the Selberg class $\mathcal{S}$, we define $d=2 \sum_{j} \lambda_{j}$ to be the degree of the function. We denote by $\mathcal{S}_{d}$ the subclass of functions with degree $d$ in the Selberg class $\mathcal{S}$. We note that the structure of $\mathcal{S}_{d}$ has been completely determined for $0 \leqslant d \leqslant 1$. From the work of Conrey and Ghosh [4], we have $\mathcal{S}_{0}=\{1\}$ and $\mathcal{S}_{d}=\emptyset$ for $0<d<1$. For $d=1$, the functions $F \in \mathcal{S}_{1}$ are of forms $F(s)=\zeta(s)$ or $F(s)=L(s+i \theta, \chi)$ with a primitive Dirichlet character $\chi$ together with $\theta \in \mathbb{R}$. On the other hand, we denote by $\mathcal{S}^{\#}$ the extended Selberg class of functions satisfying the conditions $1-3$, and we similarly define $\mathcal{S}_{d}^{\#}$ as $\mathcal{S}_{d}$. Theorems 1 and 2 in [10] describe the structure of $\mathcal{S}_{d}^{\#}$ for $0 \leqslant d \leqslant 1$.

If $d=0$, the functional equation is $Q^{s} F(s)=\omega Q^{1-s} \bar{F}(1-s)$. The proof of Theorem 3.1 [4] shows that the Dirichlet series $F(s)=\sum_{n} \frac{a(n)}{n^{s}} \in S_{0}^{\#}$ is absolutely convergent in the whole complex plane. Thus, we have

$$
\sum_{n=1}^{\infty} a(n)\left(\frac{Q^{2}}{n}\right)^{s}=\omega Q \sum_{n=1}^{\infty} \frac{\overline{a(n)}}{n} n^{s} .
$$

We let $q=Q^{2}$, then $a(n)=0$ for $n \nmid q$. For $n \mid q$, we have

$$
a(n)=\frac{\omega n}{\sqrt{q}} \overline{a\left(\frac{q}{n}\right)} .
$$

Theorem A (Theorem 3.1 of [4]). $\quad$ 1. If $0<d<1$, then $\mathcal{S}_{d}^{\#}=\emptyset$. If $F \in \mathcal{S}_{0}^{\#}$, then $q \in \mathbb{N}$, the pair $(q, \omega)$ is an invariant of $F(s)$ and $\mathcal{S}_{0}^{\#}$ is the disjoint union of the subclasses $\mathcal{S}_{0}^{\#}(q, \omega)$, with $q \in \mathbb{N}$ and $|\omega|=1$.

2. Every $F \in \mathcal{S}_{0}^{\#}(q, \omega)$, with $q$ and $\omega$ as above, is a Dirichlet polynomial of the form

$$
F(s)=\sum_{n \mid q} \frac{a(n)}{n^{s}} .
$$

For $d=1$, we use the notation

$$
\begin{aligned}
& \beta=\prod_{j=1}^{r} \lambda_{j}^{-2 \lambda_{j}}, \quad \xi=2 \sum_{j=1}^{r}\left(\mu_{j}-\frac{1}{2}\right)=\eta+i \theta, \quad q=\frac{2 \pi Q^{2}}{\beta} \\
& \omega^{*}=\omega e^{-i \pi(\eta+1) / 2}\left(\frac{Q^{2}}{\beta}\right)^{i \theta} \prod_{j=1}^{r} \lambda_{j}^{-2 i \operatorname{Im} \mu_{j}} .
\end{aligned}
$$


If $\chi$ is a Dirichlet character mod $q$, we denote by $f_{\chi}$ its conductor, and by $\chi^{*}$ the primitive character inducing $\chi$. We denote by $\omega_{\chi^{*}}$ and $Q_{\chi^{*}}$ the $\omega$-factor and the $Q$-factor in the standard functional equation for $L\left(s, \chi^{*}\right)$, i.e., $\omega_{\chi^{*}}=\tau\left(\chi^{*}\right) / i^{\mathfrak{a}} \sqrt{f_{\chi}}$, where $\tau\left(\chi^{*}\right)$ is the Gauss sum, $\mathfrak{a}=0$ if $\chi(-1)=1$ and $\mathfrak{a}=1$ if $\chi(-1)=-1$, and $Q_{\chi^{*}}=\sqrt{f_{\chi} / \pi}$. Moreover, we write

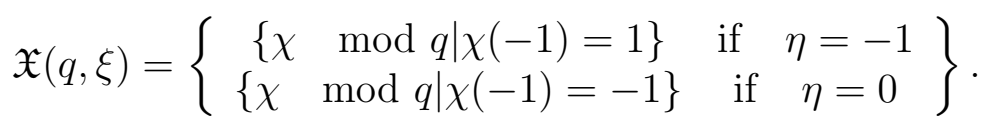

$\chi_{0}$ denotes the principal character $\bmod q$.

Theorem B (Theorem 2 of [10]). $\quad$ 1. If $F \in \mathcal{S}_{1}^{\#}$, then $q \in \mathbb{N}$ and $\eta \in\{-1,0\}$. The triple $\left(q, \xi, \omega^{*}\right)$ is an invariant of $F(s)$, and $\mathcal{S}_{1}^{\#}$ is the disjoint union of the subclasses $\mathcal{S}_{1}^{\#}\left(q, \xi, \omega^{*}\right)$ with $q \in \mathbb{N}, \eta \in\{-1,0\}, \theta \in \mathbb{R}$ and $\left|\omega^{*}\right|=1$. Moreover, $a(n) n^{i \theta}$ is periodic with period $q$.

2. Every $F \in \mathcal{S}_{1}^{\#}\left(q, \xi, \omega^{*}\right)$, with $q$, $\xi$ and $\omega^{*}$ as above, can be uniquely written as

$$
F(s)=\sum_{\chi \in \mathfrak{X}(q, \xi)} P_{\chi}(s+i \theta) L\left(s+i \theta, \chi^{*}\right)
$$

where $P_{\chi} \in \mathcal{S}_{0}^{\#}\left(q / f_{\chi}, \omega^{*} \bar{\omega}_{\chi^{*}}\right)$. Moreover, $P_{\chi_{0}}(1)=0$ if $\theta \neq 0$.

Bombieri and Hejhal [2] studied the distribution of zeros of the linear combination $F(s)=$ $\sum_{j=1}^{J} b_{j} e^{i \alpha_{j}} L_{j}(s)$ of various $L$-functions with a same gamma factor. Assuming the orthonormality condition of $a_{j}(p)$, the generalized Riemann hypothesis for $L_{j}(s)$ and a weak condition of the spacing of zeros of $L_{j}(s)$, they proved that almost all zeros of $F(s)$ are simple and on the critical line $\operatorname{Re}(s)=\frac{1}{2}$. Hejhal [7] studied the behavior of zeros of $F(s)$ near the critical line and announced that the true order of the number of zeros of $F(s)$ in $\operatorname{Re} s \geqslant \sigma$ and $T \leqslant \operatorname{Im} s \leqslant T+H$ is

$$
\frac{H}{\left(\sigma-\frac{1}{2}\right) \sqrt{\log \log T}}
$$

for $\frac{1}{2}+\frac{(\log \log T)^{\kappa}}{\log T} \leqslant \sigma \leqslant \frac{1}{2}+(\log T)^{-\delta}, c_{1} T^{w} \leqslant H \leqslant c_{2} T, \kappa>2$ with small possible exceptions of $\left\{b_{j}\right\}_{j=1}^{J}$. Note that this result for the special case $J=2$ was also justified by the same author [6].

Recently, the second author [12] investigated the off-line zeros of Epstein zeta function $E(s, Q)$ associated to quadratic form $Q(x, y)=a x^{2}+b x y+c y^{2}, a>0, b^{2}-4 a c<0$, $a, b, c \in \mathbb{Z}$. It is a classical example that belongs to the class $\mathcal{S}_{2}^{\#}$. We get the number of zeros $N_{E}\left(\sigma_{1}, \sigma_{2} ; 0, T\right)$ on the rectangular region $\sigma_{1}<\operatorname{Re} s<\sigma_{2}, 0<\operatorname{Im} s<T$ to be $c\left(\sigma_{1}, \sigma_{2}\right) T+o(T)$ for $\frac{1}{2}<\sigma_{1}<\sigma_{2}$ which improves Voronin's result [15] (or Chapter 7 of [11]) based on the joint universality theorem for Hecke $L$-functions. For $\mathcal{S}_{1}^{\#}$, Kaczorowski and Kulas [9] and Saias and Weingartner [13] proved the true order of the number of off-line zeros inside and outside the critical strip, respectively. [9] is based on the joint universality theorem for Dirichlet $L$-functions inside the critical strip and [13] is based on the idea of 
Davenport and Heilbronn [5]. The main purpose of this paper is improving their results by obtaining an asymptotic formula of $N_{F}\left(\sigma_{1}, \sigma_{2} ; 0, T\right)$ for $F \in \mathcal{S}_{1}^{\#}$ by means of the classical theory of mean motions.

By Theorems A and B, we can let the function $E(s+i \theta) \in \mathcal{S}_{1}^{\#}$ as

$$
E(s)=\sum_{j=1}^{J} h_{j}\left(p_{1}^{-s}, \ldots, p_{k}^{-s}\right) \prod_{p>p_{k}}\left(1-\frac{\chi_{j}(p)}{p^{s}}\right)^{-1}
$$

for some integer $k>0$, where

$$
h_{j}\left(x_{1}, \ldots, x_{k}\right)=\tilde{h}_{j}\left(x_{1}, \ldots, x_{k}\right) \prod_{l \leqslant k}\left(1-\chi_{j}\left(p_{l}\right) x_{l}\right)^{-1}
$$

and $\tilde{h}_{j}$ is a polynomial of $k$ variables. Let

$$
E_{n}(s)=\sum_{j=1}^{J} h_{j}\left(p_{1}^{-s}, \ldots, p_{k}^{-s}\right) \prod_{p_{k}<p \leqslant p_{n}}\left(1-\frac{\chi_{j}(p)}{p^{s}}\right)^{-1}
$$

for $n>k$. Then, $E_{n}(s)$ converges in the mean with index 2 towards $E(s)$ in $\left[\frac{1}{2}, \infty\right]$ by Parseval's identity for almost periodic functions, i.e.,

$$
\limsup _{T \rightarrow \infty} \frac{1}{T} \int_{1}^{T} \int_{\alpha}^{\beta}\left|E(\sigma+i t)-E_{n}(\sigma+i t)\right|^{2} d \sigma d t \rightarrow 0
$$

as $n \rightarrow \infty$ for any reduced strip $\frac{1}{2}<\alpha<\beta$. We may find the way of a proof in Proposition 2.3 of [12]. Applying Lemma 2.3 to $E_{n}(s)$, we get an asymptotic formula for $N_{E_{n}}\left(\sigma_{1}, \sigma_{2} ; 0, T\right)$. The theory of mean motions partially preserves this property through the convergence in the mean with index $p>0$.

If $J=1$, then we encounter with the well-known conjecture. In this paper, we only consider the case $J>1$.

We consider $\mathcal{S}_{1}^{\#}\left(p, \xi, \omega^{*}\right)$ for a prime $p$. By (1.1) and Theorem B, we have

$$
\tilde{h}_{j}=a_{j}(1) \quad \text { or } \quad a_{j}(1)+\frac{\omega \overline{a_{j}(1)}}{p^{s-\frac{1}{2}}},
$$

and as a result $\tilde{h}_{j} \neq 0$ for $\operatorname{Re} s>\frac{1}{2}$. In this case, the method in [12] works, and we have the following theorem.

Theorem 1. Let $E(s+i \theta) \in \mathcal{S}_{1}^{\#}\left(p, \xi, \omega^{*}\right)$ for $p$ prime and $|\omega|=1$, and let $\frac{1}{2}<\sigma_{1}<\sigma_{2}$. Suppose $J>1$ in (1.2). Then, we have

$$
N_{E}\left(\sigma_{1}, \sigma_{2} ; 0, T\right)=c\left(\sigma_{1}, \sigma_{2}\right) T+o(T)
$$

as $T \rightarrow \infty$. The constant $c\left(\sigma_{1}, \sigma_{2}\right)$ can be represented as an integral $\int_{\sigma_{1}}^{\sigma_{2}} H_{\sigma}(0) d \sigma$ for the density function $H_{\sigma}(x)$ of some distribution $\mu_{\sigma}$, and $c\left(\sigma_{1}, \sigma_{2}\right)>0$ if $\frac{1}{2}<\sigma_{1} \leqslant 1$. In particular, for $\sigma_{0}>\frac{1}{2}$, the number of zeros on the line segment $\operatorname{Re} s=\sigma_{0}, 0<\operatorname{Im} s<T$ is $o(T)$. 
When $q$ is a prime power, then $\tilde{h}_{j}$ are polynomials of the same single variable by Theorems $\mathrm{A}(2)$ and $\mathrm{B}(2)$. If these polynomials have the same factor with $c T+o(T)$ zeros on the line segment $\operatorname{Re} s=\sigma_{0}, 0<\operatorname{Im} s<T$ for some $\frac{1}{2}<\sigma_{0}<1$, then we cannot expect the integral form of the constant $c\left(\sigma_{1}, \sigma_{2}\right)$ in general. Indeed, we may take $\tilde{h}_{j}\left(p^{-s}\right)=1+2 p^{\frac{3}{4}-s}+p^{1-2 s}$ by letting $\omega=a(1)=1$, and $a(p)=2 p^{-\frac{3}{4}}$. Then, it has $\frac{\log p}{2 \pi} T+O(1)$ zeros on $\operatorname{Re} s=$ $\frac{\log \left(p^{\frac{3}{4}}-\sqrt{p^{\frac{3}{2}}-p}\right)}{\log p}, 0<\operatorname{Im} s<T$. We still have the following.

Theorem 2. Let $E(s+i \theta) \in \mathcal{S}_{1}^{\#}\left(q, \xi, \omega^{*}\right)$ for $q$ prime power, and let $\frac{1}{2}<\sigma_{1}<\sigma_{2}$. Suppose $J>1$ in (1.2). Then, we have

$$
N_{E}\left(\sigma_{1}, \sigma_{2} ; 0, T\right)=c\left(\sigma_{1}, \sigma_{2}\right) T+o(T)
$$

as $T \rightarrow \infty$, and $c\left(\sigma_{1}, \sigma_{2}\right)>0$ if $\frac{1}{2}<\sigma_{1} \leqslant 1$. Suppose that the closed interval $\left[\sigma_{1}, \sigma_{2}\right]$ does not contain the real part of exceptional points satisfying $h_{j}=0$. Then, the constant $c\left(\sigma_{1}, \sigma_{2}\right)$ can be represented as an integral $\int_{\sigma_{1}}^{\sigma_{2}} H_{\sigma}(0) d \sigma$ for the density function $H_{\sigma}(x)$ of some distribution $\mu_{\sigma}$. In this case for $\sigma_{0} \in\left[\sigma_{1}, \sigma_{2}\right]$, the number of zeros on the line segment $\operatorname{Re} s=\sigma_{0}, 0<\operatorname{Im} s<T$ is $o(T)$.

For general $q$, we could also prove the similar theorem, although it is not easy to classify the common zeros of $\tilde{h}_{j}$ with multiple variables. We will discuss and prove a general theorem in the section 3 .

\section{Lemmas}

We begin with the work of Jessen and Tornehave [8] that explains zeros of Dirichlet series in the region of its absolute convergence. For the basic theory of almost periodic functions, we refer to [1].

Lemma 2.1 (Theorem 8 of [8]). A function $f(s)$ almost periodic in $[\alpha, \beta]$ and not identically zero has no zeros in the sub-strip $(\alpha \leqslant) \alpha_{0}<\sigma, \beta_{0}(\leqslant \beta)$, if and only if its Jensen function $\varphi(\sigma)$ is linear in the interval $\left(\alpha_{0}, \beta_{0}\right)$.

Lemma 2.2 (Theorem 31 of [8]). For an ordinary Dirichlet series

$$
f(s)=\sum_{n=n_{0}}^{\infty} \frac{a_{n}}{n^{s}}, \quad a_{n_{0}} \neq 0
$$

with the uniform convergence abscissa $\alpha$, the Jensen function $\varphi(\sigma)$ possesses on every halfline $\sigma>\alpha_{1}(>\alpha)$ only a finite number of linearity intervals and a finite number of points of non-differentiability. The values of $\varphi^{\prime}(\sigma)$ in the linearity intervals belong to the set of numbers $-\log n, n \geqslant n_{0}$. For $\sigma>$ (some) $\sigma_{0}$, we have

$$
\varphi(\sigma)=-\left(\log n_{0}\right) \sigma+\log \left|a_{n_{0}}\right| .
$$


For an arbitrary strip $\left(\sigma_{1}, \sigma_{2}\right)$, where $\alpha<\sigma_{1}<\sigma_{2}<\infty$, the relative frequency $H\left(\sigma_{1}, \sigma_{2}\right)$ of zeros exists and is determined by

$$
H\left(\sigma_{1}, \sigma_{2}\right)=\frac{1}{2 \pi}\left(\varphi^{\prime}\left(\sigma_{2}-\right)-\varphi^{\prime}\left(\sigma_{1}+\right)\right)
$$

The following lemma guarantees the existence of second derivative of Jensen functions for almost periodic functions and gives another representation by a certain distribution. The proof can be found in $\S 9$ of [3].

Lemma 2.3 (Proposition 2.1 of [12]). Let $f(s)$ be almost periodic in the strip $[\alpha, \beta]$ and not identically zero. Let $\nu_{\sigma}$ be the asymptotic distribution function of $f(\sigma+i t)$ with respect to $\left|f^{\prime}(\sigma+i t)\right|^{2}$. Suppose $\nu_{\sigma}$ is absolutely continuous for every $\sigma$ and its density $G_{\sigma}(x)$ is a continuous function of $x$ and $\sigma$. Then the Jensen function $\varphi_{f-x}(\sigma)$ is twice differentiable with the second derivative $\varphi_{f-x}^{\prime \prime}(\sigma)=2 \pi G_{\sigma}(x)$.

The next lemma is the extension of Lemma 2.3 which is applicable inside the critical strip and which plays the main role of this method.

Lemma 2.4 (Theorem 1 of [3]). Let $-\infty \leqslant \alpha<\alpha_{0}<\beta_{0}<\beta \leqslant \infty$ and let $f_{1}(s), f_{2}(s), \ldots$ be a sequence of functions almost periodic in $[\alpha, \beta]$ converging uniformly in $\left[\alpha_{0}, \beta_{0}\right]$ towards a function $f(s)$. Suppose that none of the functions is identically zero and $f(s)$ may be continued as a regular function in the half strip $\alpha<\sigma<\beta, t>\gamma_{0}$, and that $f_{n}(s)$ converges in mean with an index $p>0$ towards $f(s)$ in $[\alpha, \beta]$. Then the Jensen function

$$
\varphi_{f}(\sigma)=\lim _{T \rightarrow \infty} \frac{1}{T} \int_{\gamma}^{T} \log |f(\sigma+i t)| d t
$$

exists uniformly in $[\alpha, \beta]$ for some $\gamma>\gamma_{0}$, and $\varphi_{f_{n}}(\sigma)$ converges uniformly in $[\alpha, \beta]$ towards $\varphi_{f}(\sigma)$ as $n \rightarrow \infty$. The function $\varphi_{f}(\sigma)$ is convex in $(\alpha, \beta)$, and for every strip $\left(\sigma_{1}, \sigma_{2}\right)$ where $\alpha<\sigma_{1}<\sigma_{2}<\beta$, the two relative frequencies of zeros defined by

$$
\begin{aligned}
& \underline{H}_{f}\left(\sigma_{1}, \sigma_{2}\right)=\liminf _{T \rightarrow \infty} \frac{1}{T} N_{f}\left(\sigma_{1}, \sigma_{2} ; \gamma, T\right), \\
& \bar{H}_{f}\left(\sigma_{1}, \sigma_{2}\right)=\limsup _{T \rightarrow \infty} \frac{1}{T} N_{f}\left(\sigma_{1}, \sigma_{2} ; \gamma, T\right),
\end{aligned}
$$

satisfy the inequalities

$$
\frac{1}{2 \pi}\left(\varphi_{f}^{\prime}\left(\sigma_{2}-\right)-\varphi_{f}^{\prime}\left(\sigma_{1}+\right)\right) \leqslant \underline{H}_{f}\left(\sigma_{1}, \sigma_{2}\right) \leqslant \bar{H}_{f}\left(\sigma_{1}, \sigma_{2}\right) \leqslant \frac{1}{2 \pi}\left(\varphi_{f}^{\prime}\left(\sigma_{2}+\right)-\varphi_{f}^{\prime}\left(\sigma_{1}-\right)\right)
$$

Suppose further that $\varphi_{f}(\sigma)$ is twice differentiable. Then, we have

$$
N_{f}\left(\sigma_{1}, \sigma_{2} ; 0, T\right)=\frac{T}{2 \pi} \int_{\sigma_{1}}^{\sigma_{2}} \varphi_{f}^{\prime \prime}(\sigma) d \sigma+o(T)
$$

for $\alpha<\sigma_{1}<\sigma_{2}<\beta$ as $T \rightarrow \infty$. 
Together with the above lemmas, we investigate the Fourier transforms of certain distributions. For our purpose, we need the two more lemmas.

We use the following notations:

$$
\begin{gathered}
\mathfrak{L}_{n}\left(\sigma, \Theta ; \chi_{j}\right)=L_{k}\left(\sigma, \theta ; \chi_{j}\right) L_{k, n}\left(\sigma, \vartheta ; \chi_{j}\right), \\
L_{k}\left(\sigma, \theta ; \chi_{j}\right)=h_{j}\left(p_{1}^{-\sigma} e^{2 \pi i \theta_{1}}, \ldots, p_{k}^{-\sigma} e^{2 \pi i \theta_{k}}\right), \\
L_{k, n}\left(\sigma, \vartheta ; \chi_{j}\right)=\prod_{k<l \leqslant n}\left(1-\frac{\chi_{j}\left(p_{l}\right) e^{2 \pi i \vartheta_{l}}}{p_{l}^{\sigma}}\right)^{-1}, \\
M_{n, \sigma}(\vartheta)=\left(\log L_{k, n}\left(\sigma, \vartheta ; \chi_{1}\right), \ldots, \log L_{k, n}\left(\sigma, \vartheta ; \chi_{J}\right)\right), \\
E_{n, \sigma}(\Theta)=\sum_{j=1}^{J} \mathfrak{L}_{n}\left(\sigma, \Theta ; \chi_{j}\right)
\end{gathered}
$$

for $n>k, \Theta=(\theta, \vartheta) \in[0,1]^{n}, \theta=\left(\theta_{1}, \ldots, \theta_{k}\right) \in[0,1]^{k}$ and $\vartheta=\left(\vartheta_{k+1}, \ldots, \vartheta_{n}\right) \in$ $[0,1]^{n-k}$. Let $\mu_{n, \sigma}$ be the distribution function of $E_{n, \sigma}$ with respect to $\left|\frac{\partial}{\partial \sigma} E_{n, \sigma}\right|^{2}$. Then, its Fourier transform is

$$
\hat{\mu}_{n, \sigma}(y)=\int_{[0,1]^{n}} e^{i \sum_{j} \mathfrak{L}_{n}\left(\sigma, \Theta ; \chi_{j}\right) \cdot y}\left|\sum_{j} \mathfrak{L}_{n}^{\prime}\left(\sigma, \Theta ; \chi_{j}\right)\right|^{2} d \Theta .
$$

Lemma 2.5. For $\sigma>\frac{1}{2}, \delta>0$ and $j \leqslant J$, we define

$$
A_{j, \sigma}(\delta)=\left\{\theta \in[0,1]^{k}:\left|\tilde{h}_{j}\left(p_{1}^{-\sigma} e^{2 \pi i \theta_{1}}, \ldots, p_{k}^{-\sigma} e^{2 \pi i \theta_{k}}\right)\right|<\delta\right\} .
$$

Then, for any integer $K \leqslant J$ we have

$$
\hat{\mu}_{n, \sigma}(y) \ll\left|\bigcap_{r_{1}<\cdots<r_{K} \leqslant J}\left(A_{r_{1}, \sigma}(\delta) \cup \cdots \cup A_{r_{K}, \sigma}(\delta)\right)\right|+|\delta y|^{-K}
$$

as $|y| \rightarrow \infty$, where the corresponding constant does not depend on $n$.

Proof. We write

$$
\hat{\mu}_{n, \sigma}(y)=\sum_{l_{1}, l_{2}} \int_{[0,1]^{n}} e^{i \sum_{j} \mathfrak{L}_{n}\left(\sigma, \Theta ; \chi_{j}\right) \cdot y} \mathfrak{L}_{n}^{\prime}\left(\sigma, \Theta ; \chi_{l_{1}}\right) \overline{\mathfrak{L}_{n}^{\prime}\left(\sigma, \Theta ; \chi_{l_{2}}\right)} d \Theta .
$$

Define set functions

$$
\begin{aligned}
\lambda_{n, \sigma ; l_{1}, l_{2}}(B) & =\int_{M_{n, \sigma}^{-1}(B)} \frac{L_{k, n}^{\prime}}{L_{k, n}}\left(\sigma, \vartheta ; \chi_{l_{1}}\right) \overline{\frac{L_{k, n}^{\prime}}{L_{k, n}}\left(\sigma, \vartheta ; \chi_{l_{2}}\right)} d \vartheta \\
\lambda_{n, \sigma ; l}(B) & =\int_{M_{n, \sigma}^{-1}(B)} \frac{L_{k, n}^{\prime}}{L_{k, n}}\left(\sigma, \vartheta ; \chi_{l}\right) d \vartheta \\
\lambda_{n, \sigma}(B) & =\left|M_{n, \sigma}^{-1}(B)\right|
\end{aligned}
$$


for any Borel sets $B \subset \mathbb{C}^{J}$. By the identity

$$
a \bar{b}=\frac{1}{4} \sum_{m=1}^{4} i^{m}\left|a+i^{m} b\right|^{2} \quad a, b \in \mathbb{C},
$$

one can prove that $\hat{\mu}_{n, \sigma}(y)$ is a linear combination of at most four absolutely continuous distribution functions. (See [12] for details.) Thus, we denote corresponding densities $G_{n, \sigma ; l_{1}, l_{2}}(x)$, $G_{n, \sigma ; l}(x), G_{n, \sigma}(x)$ of $\lambda_{n, \sigma ; l_{1}, l_{2}}, \lambda_{n, \sigma ; l}, \lambda_{n, \sigma}$, respectively. By Theorem 6 of [3], the densities $G_{n, \sigma ; l_{1}, l_{2}}(x), G_{n, \sigma ; l}(x), G_{n, \sigma}(x)$ have a majorant of the form $K e^{-\lambda|x|^{2}}$, and their partial derivatives of order $\leqslant q$ have a majorant of the form $K_{q} e^{-\lambda|x|^{2}}$ for $n \geqslant n_{q}$. Thus, we have

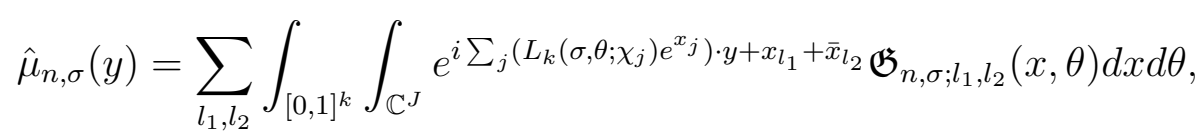

where

$$
\begin{aligned}
\mathfrak{G}_{n, \sigma ; l_{1}, l_{2}}(x, \theta) & =L_{k}^{\prime}\left(\sigma, \theta ; \chi_{l_{1}}\right) \overline{L_{k}^{\prime}\left(\sigma, \theta ; \chi_{l_{2}}\right)} G_{n, \sigma}(x)+L_{k}^{\prime}\left(\sigma, \theta ; \chi_{l_{1}}\right) \overline{L_{k}\left(\sigma, \theta ; \chi_{l_{2}}\right) G_{n, \sigma ; l_{2}}(x)} \\
& +L_{k}\left(\sigma, \theta ; \chi_{l_{1}}\right) \overline{L_{k}^{\prime}\left(\sigma, \theta ; \chi_{l_{2}}\right)} G_{n, \sigma ; l_{1}}(x)+L_{k}\left(\sigma, \theta ; \chi_{l_{1}}\right) \overline{L_{k}\left(\sigma, \theta ; \chi_{l_{2}}\right)} G_{n, \sigma ; l_{1}, l_{2}}(x) .
\end{aligned}
$$

We only consider the first term $L_{k}^{\prime}\left(\sigma, \theta ; \chi_{l_{1}}\right) \overline{L_{k}^{\prime}\left(\sigma, \theta ; \chi_{l_{2}}\right)} G_{n, \sigma}(x)$, since the others can be treated similarly. If $\theta \notin A_{j, \sigma}(\delta)$ for $K$-many $j$, integration by parts with respect to such $x_{j}$ gives

$$
\int_{\mathbb{C}^{J}} e^{i \sum_{j}\left(L_{k}\left(\sigma, \theta ; \chi_{j}\right) e^{x_{j}}\right) \cdot y+x_{l_{1}}+\bar{x}_{l_{2}}} G_{n, \sigma}(x) d x=O\left(|\delta y|^{-K}\right) .
$$

For the other $\theta$, we give a trivial upperbound by its measure, and we have

$$
\hat{\mu}_{n, \sigma}(y) \ll\left|\bigcap_{r_{1}<\cdots<r_{K} \leqslant J}\left(A_{r_{1}, \sigma}(\delta) \cup \cdots \cup A_{r_{K}, \sigma}(\delta)\right)\right|+|\delta y|^{-K},
$$

where the corresponding constant does not depend on $n$ as $y \rightarrow \infty$.

So, it is enough to show the equation (2.1). We decompose

$$
\begin{aligned}
& \int_{\mathbb{C}^{J}} e^{i \sum_{j}\left(L_{k}\left(\sigma, \theta ; \chi_{j}\right) e^{x_{j}}\right) \cdot y+x_{l_{1}}+\bar{x}_{l_{2}} G_{n, \sigma}(x) d x} \\
= & \sum_{m \in \mathbb{Z}^{J}} \int_{(\mathbb{R} \times[0,2 \pi])^{J}} e^{i \sum_{j} e^{x_{j}} \cdot\left(\overline{L_{k}\left(\sigma, \theta ; \chi_{j}\right)} y\right)+x_{l_{1}}+\bar{x}_{l_{2}}} G_{n, \sigma}(x+2 \pi m i) d x .
\end{aligned}
$$

Changing of variables $e^{x_{j}}=r_{j} e^{z_{j}}$ with Jacobian $r_{j}^{-1}$ gives

$=\sum_{m \in \mathbb{Z}^{J}} \int_{[0,2 \pi]^{J}} \int_{(0, \infty)^{J}} e^{i \sum_{j} r_{j} e^{z_{j}} \cdot\left(\overline{L_{k}\left(\sigma, \theta ; \chi_{j}\right)} y\right)+z_{l_{1}}-z_{l_{2}}} r_{l_{1}} r_{l_{2}} \prod_{j} r_{j}^{-1} G_{n, \sigma}(\log r+i(z+2 \pi m)) d r d z$, 
where $r=\left(r_{1}, \ldots, r_{J}\right), z=\left(z_{1}, \ldots, z_{J}\right)$, and $\log r=\left(\log r_{1}, \ldots, \log r_{J}\right)$. Consider the integration

$$
\begin{aligned}
& \int_{0}^{2 \pi} \int_{0}^{\infty} e^{i r_{j} e^{z_{j}} \cdot\left(\overline{L_{k}\left(\sigma, \theta ; \chi_{j}\right)} y\right)+z_{l_{1}}-z_{l_{2}}} r_{l_{1}} r_{l_{2}} r_{j}^{-1} G_{n, \sigma}(\log r+i(z+2 \pi m)) d r_{j} d z_{j} \\
& =\int_{0}^{2 \pi} \int_{0}^{\infty} e^{i r_{j}\left|L_{k}\left(\sigma, \theta ; \chi_{j}\right) y\right| \cos \left(z_{j}-\alpha_{j}\right)+z_{l_{1}}-z_{l_{2}}} r_{l_{1}} r_{l_{2}} r_{j}^{-1} G_{n, \sigma}(\log r+i(z+2 \pi m)) d r_{j} d z_{j}
\end{aligned}
$$

for some $\alpha_{j}$. For $\theta \notin A_{j, \sigma}(\delta)$, we integrate by parts with respect to $z_{j}$ for $\left|\cos \left(z_{j}-\alpha_{j}\right)\right|<\frac{1}{2}$, and with respect to $r_{j}$ for $\left|\cos \left(z_{j}-\alpha_{j}\right)\right|>\frac{1}{2}$. With the uniform upperbound $K_{q} e^{-\lambda|x|^{2}}$ of partial derivatives of $G$ of order $\leqslant q$, we have the equation (2.1).

Lemma 2.6. $\hat{\mu}_{n, \sigma}(y)$ converges uniformly for every disc $|y| \leqslant a$ and $\frac{1}{2}<\sigma_{1} \leqslant \sigma \leqslant \sigma_{2}$

Proof. By definition, we have

$$
\hat{\mu}_{n+1, \sigma}(y)=\int_{[0,1]^{n}} \int_{0}^{1} e^{i E_{n+1, \sigma}(\Theta, u) \cdot y}\left|\frac{\partial}{\partial \sigma} E_{n+1, \sigma}(\Theta, u)\right|^{2} d u d \Theta .
$$

We get

$$
\begin{aligned}
& \int_{0}^{1} e^{i E_{n+1, \sigma}(\Theta, u) \cdot y}\left|\frac{\partial}{\partial \sigma} E_{n+1, \sigma}(\Theta, u)\right|^{2} d u=\int_{0}^{1} e^{i E_{n+1, \sigma}(\Theta, u) \cdot y} d u\left|\frac{\partial}{\partial \sigma} E_{n, \sigma}(\Theta)\right|^{2} \\
+ & \int_{0}^{1} e^{i E_{n+1, \sigma}(\Theta, u) \cdot y} 2 \operatorname{Re}\left[\frac{\partial}{\partial \sigma} \overline{E_{n, \sigma}(\Theta)} e^{2 \pi i u} \frac{\partial}{\partial \sigma} \sum_{j=1}^{J} h_{j}(\ldots) \prod_{k<j \leqslant n}(\ldots)^{-1} \frac{\chi_{j}\left(p_{n+1}\right)}{p_{n+1}^{\sigma}}\right] d u \\
+ & O\left(\frac{F_{n}(\sigma, \Theta)^{2}}{p_{n+1}^{2 \sigma}}\right)
\end{aligned}
$$

where

$$
F_{n}(\sigma, \Theta)=\sum_{j=1}^{J} \prod_{k<l \leqslant n}\left|1-\frac{\chi_{j}\left(p_{l}\right)}{p_{l}^{\sigma}} e^{2 \pi i \vartheta_{l}}\right|^{-1} .
$$

By $e^{i x}=1+i x+O\left(|x|^{2}\right)(x \in \mathbb{R})$, we have

$$
\begin{aligned}
& \int_{0}^{1} e^{i E_{n+1, \sigma}(\Theta, u) \cdot y} d u \\
= & \int_{0}^{1} e^{i E_{n, \sigma}(\Theta) \cdot y}\left(1+i\left(E_{n+1, \sigma}(\Theta, u)-E_{n, \sigma}(\Theta)\right) \cdot y\right) d u+O\left(\frac{F_{n}(\sigma, \Theta)^{2}}{p_{n+1}^{2 \sigma}}\right) \\
= & e^{i E_{n, \sigma}(\Theta) \cdot y}+O\left(\frac{F_{n}(\sigma, \Theta)^{2}}{p_{n+1}^{2 \sigma}}\right) .
\end{aligned}
$$


By $e^{i x}=1+O(|x|)(x \in \mathbb{R})$, we have

$$
\begin{aligned}
\int_{0}^{1} e^{i E_{n+1, \sigma}(\Theta, u) \cdot y \pm 2 \pi i u} d u & =\int_{0}^{1} e^{i E_{n, \sigma}(\Theta) \cdot y \pm 2 \pi i u} d u+O\left(\frac{F_{n}(\sigma, \Theta)}{p_{n+1}^{\sigma}}\right) \\
& =O\left(\frac{F_{n}(\sigma, \Theta)}{p_{n+1}^{\sigma}}\right) .
\end{aligned}
$$

Combining the above equations yields

$$
\begin{aligned}
\int_{0}^{1} e^{i E_{n+1, \sigma}(\Theta, u) \cdot y}\left|\frac{\partial}{\partial \sigma} E_{n+1, \sigma}(\Theta, u)\right|^{2} d u & =e^{i E_{n, \sigma}(\Theta) \cdot y}\left|\frac{\partial}{\partial \sigma} E_{n, \sigma}(\Theta)\right|^{2} \\
& +O\left(\frac{F_{n}(\sigma, \Theta)^{2}+F_{n}(\sigma, \Theta)^{3}+F_{n}(\sigma, \Theta)^{4}}{p_{n+1}^{2 \sigma}} \log p_{n+1}\right) .
\end{aligned}
$$

Thus, we have

$$
\hat{\mu}_{n+1, \sigma}(y)-\hat{\mu}_{n, \sigma}(y)=O\left(p_{n+1}^{-2 \sigma} \log p_{n+1}\right)
$$

and

$$
\hat{\mu}_{m, \sigma}(y)-\hat{\mu}_{n, \sigma}(y)=O\left(p_{n}^{1-2 \sigma_{1}}\right)
$$

for $m>n>k$. Thus, Lemma 2.6 follows.

\section{Main results}

We divide the cases $J=2$ and $J \geqslant 3$. For $J=2$, our function is the sum of two spoiled Euler products $f_{1}(s)+f_{2}(s)$. We then apply the theory of value distribution for $f_{1}(s)$ and $\frac{f_{2}}{f_{1}}(s)$.

Proposition 3.1. Let $J=2$ and $\frac{1}{2}<\sigma_{1}<\sigma_{2}$. Suppose that $h_{j}\left(p_{1}^{-\sigma} e^{2 \pi i \theta_{1}}, \ldots, p_{k}^{-\sigma} e^{2 \pi i \theta_{k}}\right) \neq 0$ for $j=1,2, \sigma_{1} \leqslant \sigma \leqslant \sigma_{2}$, and $\theta \in[0,1]^{k}$. Then, we have

$$
N_{E}\left(\sigma_{1}, \sigma_{2} ; 0, T\right)=T \int_{\sigma_{1}}^{\sigma_{2}} H_{\sigma}(-1) d \sigma+o(T),
$$

where $H_{\sigma}(x)$ is the density for some distribution function $\mu_{\sigma}$. Moreover, $H_{\sigma}(x)>0$ for $\frac{1}{2}<\sigma \leqslant 1$.

Proof. By Lemma 2.4, $\varphi_{E_{n}}(\sigma)$ converges uniformly to $\varphi_{E}(\sigma)$ on $\left[\frac{1}{2}, \infty\right)$. If $\varphi_{E}(\sigma)$ is twice differentiable, then we have

$$
N_{E}\left(\sigma_{1}, \sigma_{2} ; 0, T\right)=\frac{T}{2 \pi} \int_{\sigma_{1}}^{\sigma_{2}} \varphi_{E}^{\prime \prime}(\sigma) d \sigma+o(T) .
$$

By direct calculation, we have

$$
\varphi_{E_{n}}(\sigma)=\varphi_{h_{2}}(\sigma)+\varphi_{\tilde{L}_{n}+1}(\sigma)
$$


where

$$
\tilde{L}_{n}(s)=\frac{h_{1}}{h_{2}}\left(p_{1}^{-s}, \ldots, p_{k}^{-s}\right) \prod_{p_{k}<p \leqslant p_{n}} \frac{1-\frac{\chi_{2}(p)}{p^{s}}}{1-\frac{\chi_{1}(p)}{p^{s}}} .
$$

By Lemma 2.1, we have $\varphi_{h_{2}}^{\prime \prime}(\sigma)=0$ for $\sigma_{1} \leqslant \sigma \leqslant \sigma_{2}$. For $\tilde{L}_{n}$, the method in chapter II in [3] works. Define

$$
\begin{gathered}
\tilde{L}_{n, \sigma}(\Theta)=\frac{h_{1}}{h_{2}}\left(p_{1}^{-\sigma} e^{2 \pi i \theta_{1}}, \ldots, p_{k}^{-\sigma} e^{2 \pi i \theta_{k}}\right) \prod_{k<l \leqslant n} \frac{1-\frac{\chi_{2}\left(p_{l}\right) e^{2 \pi i \vartheta_{l}}}{p_{l}^{\sigma}}}{1-\frac{\chi_{1}\left(p_{l}\right) e^{2 \pi i \vartheta_{l}}}{p_{l}^{\sigma}}}, \\
\mu_{n, \sigma}(B)=\int_{\tilde{L}_{n, \sigma}^{-1}(B)}\left|\frac{\partial}{\partial \sigma} \tilde{L}_{n, \sigma}(\Theta)\right|^{2} d \Theta
\end{gathered}
$$

for any Borel set $B \subset \mathbb{C}$ and $n>k, \Theta=\left(\theta_{1}, \ldots, \theta_{k}, \vartheta_{k+1}, \ldots, \vartheta_{n}\right) \in[0,1]^{n}$. Applying Theorems 5-10 in [3] with some modification, we prove that the absolutely continuous distributions $\mu_{n, \sigma}$ converge to a distribution $\mu_{\sigma}$ with a density $H_{\sigma}(x)$ and $\varphi_{\tilde{L}_{n}+1}^{\prime \prime}(\sigma)=2 \pi H_{\sigma}(-1)>0$ for $\frac{1}{2}<\sigma \leqslant 1$.

For the case $J \geqslant 3$, we cannot do the same thing as $J=2$. However, by virtue of the method in [12], we obtain the following.

Proposition 3.2. Let $J \geqslant 3$ and $\frac{1}{2}<\sigma_{1}<\sigma_{2}$. Suppose that $h_{j}\left(p_{1}^{-\sigma} e^{2 \pi i \theta_{1}}, \ldots, p_{k}^{-\sigma} e^{2 \pi i \theta_{k}}\right) \neq 0$ for $j=l_{1}, l_{2}, l_{3}, \sigma_{1} \leqslant \sigma \leqslant \sigma_{2}$, and $\theta \in[0,1]^{k}$. Then, we have

$$
N_{E}\left(\sigma_{1}, \sigma_{2} ; 0, T\right)=T \int_{\sigma_{1}}^{\sigma_{2}} H_{\sigma}(0) d \sigma+o(T),
$$

where $H_{\sigma}(x)$ is the density for some distribution function $\mu_{\sigma}$.

Proof. By Lemma 2.4, $\varphi_{E_{n}}(\sigma)$ converges uniformly to $\varphi_{E}(\sigma)$ on $\left[\frac{1}{2}, \infty\right)$. If $\varphi_{E}(\sigma)$ is twice differentiable, then we have

$$
N_{E}\left(\sigma_{1}, \sigma_{2} ; 0, T\right)=\frac{T}{2 \pi} \int_{\sigma_{1}}^{\sigma_{2}} \varphi_{E}^{\prime \prime}(\sigma) d \sigma+o(T) .
$$

By Lemma 2.5 with

$$
\delta=\min \left\{\mid \tilde{h}_{j}\left(p_{1}^{-\sigma} e^{2 \pi i \theta_{1}}, \ldots, p_{k}^{-\sigma} e^{2 \pi i \theta_{k}}\right) \| j=l_{1}, l_{2}, l_{3}, \sigma_{1} \leqslant \sigma \leqslant \sigma_{2}, \theta \in[0,1]^{k}\right\}>0,
$$

we have $\hat{\mu}_{n, \sigma}(y) \ll|y|^{-3}$ and this implies that $\mu_{n, \sigma}$ is absolutely continuous and its density $H_{n, \sigma}(x)$ is continuous. Let $\nu_{n, \sigma}$ be the asymptotic distribution of $E_{n}(\sigma+i t)$ with respect to $\left|E_{n}^{\prime}(\sigma+i t)\right|^{2}$. Since $\hat{\mu}_{n, \sigma}(y)=\hat{\nu}_{n, \sigma}(y)$ by Kronecker's theorem, $\mu_{n, \sigma}=\nu_{n, \sigma}$ and $H_{n, \sigma}$ is their common density. By Lemma 2.3, $\varphi_{E_{n}-x}^{\prime \prime}(\sigma)=2 \pi H_{n, \sigma}(x)$. By Lemma 2.6, $H_{n, \sigma}(x)$ 
converges to $H_{\sigma}(x)$ which is the density of some distribution $\mu_{\sigma}=\lim _{n \rightarrow \infty} \mu_{n, \sigma}$. Therefore, we have

$$
N_{E}\left(\sigma_{1}, \sigma_{2} ; 0, T\right)=T \int_{\sigma_{1}}^{\sigma_{2}} H_{\sigma}(0) d \sigma+o(T)
$$

By Lemma 2.2, each Dirichlet polynomial $h_{j}\left(p_{1}^{-s}, \ldots, p_{k}^{-s}\right)$ has at most finite number of linearity intervals of its Jensen function $\varphi_{h_{j}}(\sigma)$ in $\left[\frac{1}{2}, \infty\right)$ and say $\mathfrak{I}_{j}$ the union of the intervals. By Lemmas 2.3 and 2.4 and almost periodicity, it means that $h_{j}$ has no zero in $\mathfrak{I}_{j}$. We let $\varsigma_{j}=$ $\inf \mathfrak{I}_{j} \geqslant \frac{1}{2}$, and $\varsigma_{E}$ be the third small $\varsigma_{j}$, more precisely, $\varsigma_{E}=\varsigma_{l_{3}}$ when $\varsigma_{l_{1}} \leqslant \varsigma_{l_{2}} \leqslant \varsigma_{l_{3}} \leqslant \cdots$ is the linear order of $\varsigma_{1}, \ldots, \varsigma_{J}$. By combining Lemma 2.4 and Proposition 3.2, we have the following theorem.

Theorem 3. Let $J \geqslant 3$ and $\varsigma_{E}<\sigma_{1}<\sigma_{2}$. Suppose that $\sigma_{1}, \sigma_{2} \in \mathfrak{I}_{j}$ for at least three $j$. Then, we have

$$
N_{E}\left(\sigma_{1}, \sigma_{2} ; 0, T\right)=\frac{T}{2 \pi}\left(\varphi_{E}^{\prime}\left(\sigma_{2}-\right)-\varphi_{E}^{\prime}\left(\sigma_{1}+\right)\right)+o(T) .
$$

Suppose further that $\left[\sigma_{1}, \sigma_{2}\right] \subset \mathfrak{I}_{j}$ for at least three $j$. Then, we have

$$
N_{E}\left(\sigma_{1}, \sigma_{2} ; 0, T\right)=T \int_{\sigma_{1}}^{\sigma_{2}} H_{\sigma}(0) d \sigma+o(T)
$$

where $H_{\sigma}(x)$ is the density for some distribution $\mu_{\sigma}$. In this case, for $\sigma_{1}<\sigma_{0}<\sigma_{2}$, the number of zeros of $E(s)$ on the line segment $\operatorname{Re} s=\sigma_{0}$ and $0<\operatorname{Im} s<T$ is $o(T)$.

If each $\tilde{h}_{j}$ is non-vanishing on $\operatorname{Re} s>\frac{1}{2}$, Theorem 3 holds.

Theorem 4. Let $J \geqslant 3$ and $\frac{1}{2}<\sigma_{1}<\sigma_{2}$. Suppose that $\tilde{h}_{j} \neq 0$ for $\operatorname{Re} s>\frac{1}{2}$. Then, we have

$$
N_{E}\left(\sigma_{1}, \sigma_{2} ; 0, T\right)=T \int_{\sigma_{1}}^{\sigma_{2}} H_{\sigma}(0) d \sigma+o(T)
$$

where $H_{\sigma}(x)$ is the density for a distribution $\mu_{\sigma}$. For $\sigma_{0}>\frac{1}{2}$, the number of zeros of $E(s)$ on the line segment $\operatorname{Re} s=\sigma_{0}$ and $0<\operatorname{Im} s<T$ is $o(T)$.

As a consequence, we prove Theorem 1 .

We consider the case $\tilde{h}_{j}$ is one variable polynomial. In this case, $\tilde{h}_{j}\left(p^{-s}\right)=0$ if and only if $p^{-s}=\beta_{1}, \ldots, \beta_{m}$ for some $m$. Thus, each line segment $\operatorname{Re} s=-\frac{\log \left|\beta_{j}\right|}{\log p}$ and $0<\operatorname{Im} s<T$ contains $c T+O(1)$ zeros of $\tilde{h}_{j}\left(p^{-s}\right)$. So, we may not have the equation (3.1) for $E(s)$. However, if we disregard these exceptional points, we have the following theorem. 
Theorem 5. Let $J \geqslant 3$ and $\frac{1}{2}<\sigma_{1}<\sigma_{2}$. Let $E(s)=\sum_{j \leqslant J} \tilde{h}_{j}\left(p_{1}^{-s}, \ldots, p_{k}^{-s}\right) L\left(s, \chi_{j}\right)$, where each $\tilde{h}_{j}$ is a polynomial of one variable. Then, we have

$$
N_{E}\left(\sigma_{1}, \sigma_{2} ; 0, T\right)=\frac{T}{2 \pi}\left(\varphi_{E}^{\prime}\left(\sigma_{2}-\right)-\varphi_{E}^{\prime}\left(\sigma_{1}+\right)\right)+o(T) .
$$

Let $\mathfrak{I}=\bigcup_{l_{1}<l_{2}<l_{3} \leqslant J}\left(I_{l_{1}} \cap I_{l_{2}} \cap I_{l_{3}}\right)$ is $\left(\frac{1}{2}, \infty\right)$ except finitely many points. If $\left[\sigma_{1}, \sigma_{2}\right] \subset \mathfrak{I}$, then we have

$$
N_{E}\left(\sigma_{1}, \sigma_{2} ; 0, T\right)=T \int_{\sigma_{1}}^{\sigma_{2}} H_{\sigma}(0) d \sigma+o(T)
$$

where $H_{\sigma}(x)$ is the density for some distribution $\mu_{\sigma}$. For $\sigma_{0} \in \mathfrak{I}$, the number of zeros of $E(s)$ on the line segment $\operatorname{Re} s=\sigma_{0}$ and $0<\operatorname{Im} s<T$ is $o(T)$.

As a consequence, we prove Theorem 2 .

\section{References}

[1] A.S. Besicovitch, Almost Periodic Functions, Dover, New York, 1954.

[2] E. Bombieri and D. Hejhal, On the distribution of zeros of linear combinations of Euler products, Duke Math. J. 80 (1995), 821-862.

[3] V. Borchsenius and B. Jessen, Mean Motions and values of the Riemann zeta function, Acta Math. 80 (1948), 97-166.

[4] J.B. Conrey and A. Ghosh, On the Selberg class of Dirichlet series: small degrees, Duke Math. 72 (1993), 673-693.

[5] H. Davenport and H. Heilbronn, On the zeros of certain Dirichlet series, J. London Math. Soc. 11 (1936), 181-185, 307-312.

[6] D. Hejhal, On a result of Selberg concerning zeros of linear combinations of L-functions, Int. Math. Res. Not. (2000), 551-577.

[7] On the horizontal distribution of zeros of linear combinations of Euler products, C. R. Acad. Sci. Paris, Ser. I 338 (2004), 755-758.

[8] B. Jessen and H. Tornehave, Mean motions and zeros of almost periodic functions, Acta Math. 77 (1945), 137-279.

[9] J. Kaczorowski and M. Kulas, On the non-trivial zeros off the critical line for L-functions from the extended Selberg class, Monatsh. Math. 150 (2007), 217-232.

[10] J. Kaczorowski and A. Perelli, On the structure of the Selberg class, $I: 0 \leqslant d \leqslant 1$, Acta Math. 182 (1999), 207-241. 
[11] A.A. Karatsuba and S.M. Voronin, The Riemann Zeta-Function, Berlin, New York: Walter de Gruyter, 1992.

[12] Y. Lee, On the Zeros of Epstein Zeta Functions, preprint

[13] E. Saias and A. Weingartner, Zeros of Dirichlet series with periodic coefficients, Acta Arith. 140 (2009), 335-344.

[14] A. Selberg, "Old and new conjectures and results about a class of Dirichlet series" in Collected Papers, Vol. 2, Springer, Berlin, 1991, pp. 47-63.

[15] S.M. Voronin, The zeros of zeta-functions of quadratic forms. (Russian), Trudy Mat. Inst. Steklov. 142 (1976), 135-147.

Department of mathematics, Yonsei University, Seoul, 120-749, Korea

Korea Institute for Advanced Study, 207-43 Cheongnyangni-dong, Dongdaemun-gu, Seoul 130-722, Korea

E-mail address : Haseo Ki ( haseo@yonsei.ac.kr )

Department of mathematics, POSTECH, Pohang, Gyungbuk 790-784, Korea

E-mail address : Yoonbok Lee ( leeyb131@gmail.com ) 